\title{
Injuries sustained while surfboard riding
}

\author{
Simon Dimmick • Patrick Sheehan • David Brazier • \\ Suzanne E. Anderson
}

Received: 15 October 2012 / Accepted: 15 October 2012 /Published online: 20 November 2012

(C) ISS 2012

\section{Introduction}

The origins of surfboard riding are proposed to be in ancient Polynesia, where it was fundamental in the culture. Owing to Western colonization and influence, surfing all but disappeared, but was revived by the Hawaiians at Waikiki at the beginning of the twentieth century [1]. Surfing was introduced to California in 1907 by George Freeth and to Australia in 1915 by Duke Kahanamoku, a Hawaiian Olympic champion swimmer [1]. Today, surfboard riding is a popular recreational activity and sport worldwide, particularly in the USA and Australia. Between 5 and 23 million people worldwide participate in surfboard riding [2].

There has been significant evolution of surfboard and surfing equipment over the past 15 years. Boards are lighter, shorter, and more maneuverable. As a result, the "tricks" performed by surfers have become more complicated and potentially injurious.

Two recent retrospective studies have been undertaken at Royal North Shore Hospital (Sydney, Australia) to assess the spectrum of injuries sustained to the head and spine while surfboard riding [3]. These results have also been presented at the Asian Oceanian Congress of Radiology in Sydney in August 2012.

S. Dimmick $(\bowtie) \cdot$ P. Sheehan $\cdot$ D. Brazier

Department of Radiology, Royal North Shore Hospital,

St Leonards, 2065 NSW, Australia

e-mail: sdimmick@gmp.usyd.edu.au

S. Dimmick $\cdot$ P. Sheehan $\cdot$ S. E. Anderson

School of Medicine Sydney,

The University of Notre Dame,

Darlinghurst, NSW 2010, Australia

\section{S. E. Anderson}

Musculoskeletal Radiology, Radiology Department,

Monash Southern Health,

Clayton, Vic 3168, Australia

\section{Injuries sustained while surfing}

Although surfing is a relatively safe pastime, injuries do occur. The reported incidence of injury ranges between 2.2 to 3.5 and 6.6 injuries per 1,000 hours of surfing in recreational and competitive surfers, respectively [4-6].

\section{Acute head and spinal injuries}

The most catastrophic injuries are to the head and spine, particularly the facial region, globes, and the cervical spine. Injuries to the head and neck constitute $25-37 \%$ of all surfing injuries [4-6].

The most common mechanism of injury to the spine involves the surfer's head striking the sea floor [3]. Understandably, this results primarily in injuries to the cervical spine; however, rarely, retained surfboard components may present with delay as a foreign body, a "surfinoma" [7]. A multitude of different injuries occur in the cervical spine and depend on the position the surfer is in when they strike the sea floor relative to the force vector of the wave. Flexion, extension, axial load, rotation, and a variety of different combinations may result in fractures, dislocations, or spinal cord injuries (predominantly contusions) [3].

Contusions of the cervical cord may occur in isolation [3] and, understandably, occur in people with predisposing factors, such as congenital canal stenosis, intervertebral disc protrusion, and/or uncovertebral joint osteophyte formation.

Fractures of the thoracic and lumbar spine are less common, and are compression fractures (due to an axial load and/or flexion) or transverse process fractures of the lumbar spine.

The majority of head injuries are caused by the surfer's head striking a surfboard, mostly their own. This results predominantly in injuries to the facial region, including contusions, lacerations, and fractures. It is uncommon to sustain intra- or extra-axial hematomas due to surfingrelated trauma. 


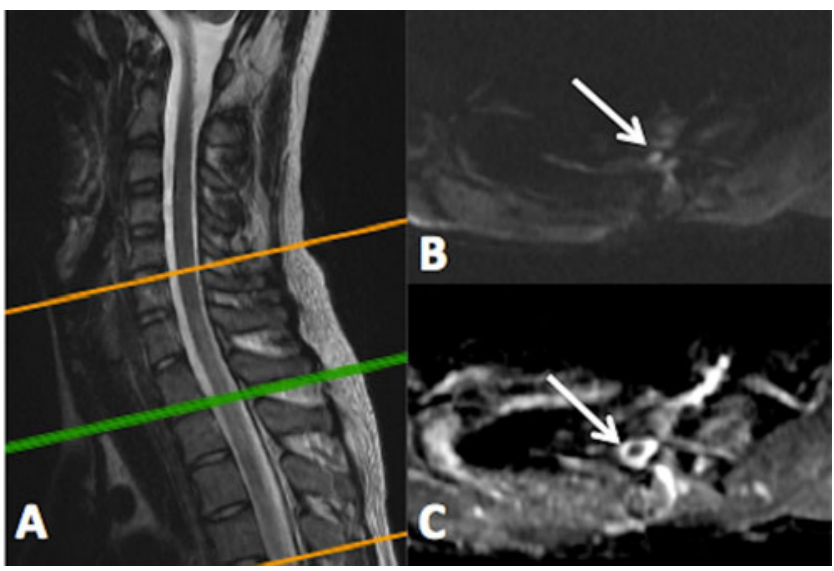

Fig. 1 Magnetic resonance images of the cervical and upper thoracic spine in an 18-year-old man who suffered progressively increasing pain and paresis clinically to the level of C7. a Sagittal T2-weighted image demonstrating extensive T2 hyperintensity within the visualized upper thoracic cord to the level of C7. Diffusion weighted image (b) at the level of T2 (denoted by the green reference line in $a$ ) and the corresponding apparent diffusion coefficient image- ADC. (c) Acute ischemia of the cord, which is hyperintense on diffusion and correspondingly hypointense on the ADC image (arrows)

Injuries to the globe may be catastrophic and may be secondary to penetrating contact with the tip or "nose" of the surfboard. More commonly, rupture of the globe is due to blunt trauma from the board.

\section{Surfer's myelopathy}

Surfer's myelopathy is an uncommon pathology that occurs in novice surfers only, who are undertaking their first surfing lesson $[3,8]$. Twenty-one cases of surfer's myelopathy have been described in the literature [3,8-16]. These people develop progressive bilateral lower limb pain, paresthesia, and paraplegia during their first lesson. Magnetic resonance imaging imaging demonstrates $\mathrm{T} 2$ /proton density hyperintensity within the inferior aspect of the cord. The exact cause of surfer's myelopathy is unknown, but an underlying predisposition may be immaturity of the erector spinae musculature. Postulated etiologies include arterial ischemia, venous hypertension causing venous ischemia, or fibrocartilaginous embolization $[8,9]$. In all documented cases of surfer's myelopathy, spinal angiography is normal. In most circumstances, these patients demonstrate variable recovery over a period of weeks to months.

However, rarely (and sadly), there may be little recovery, as demonstrated in an 18-year-old male who had a complete C7 spinal level and had extensive signal abnormality throughout the cord from $\mathrm{C} 7$ to the conus, with restricted diffusion on diffusion-weighted imaging and hemorrhage inferiorly within the cord (Figs. 1 and 2).

\section{Safety equipment}

Safety equipment has been designed for surfers, which includes helmets, nose guards that are attached to the pointed tip of the surfboard, and eyewear. To date, there have been no studies to assess the validity of these devices for the prevention of injuries. In addition, surfers have not embraced this equipment, which is considered restrictive (in the case of helmets and eyewear), and reduces maneuverability and increases resistance in the water (nose guards).

Leg ropes are one accessory that has been universally accepted universally by surfers. A leg rope (or leash) attaches the surfer's ankle to the tail of the surfboard. This device prevents the surfer from being separated from their board after a "wipeout". These leg ropes prevent injuries in two ways - when a surfer is injured they remain in close proximity to their board to provide a floatation device to get them back to the beach, and also prevent a surfer from being struck by a fellow surfer's board. However there is an increased risk of being struck by the surfer's own board. The evolution of leg ropes has optimized both the length and elastic recoil to prevent injury.

\section{Conclusions}

Although surfboard riding is a relatively safe sport, knowledge of the spectrum of injuries, their imaging characteristics, and predisposing factors is essential. With this information, technical innovations in surfboard and surfing accessory design, and public health advice to minimize the risk of injury

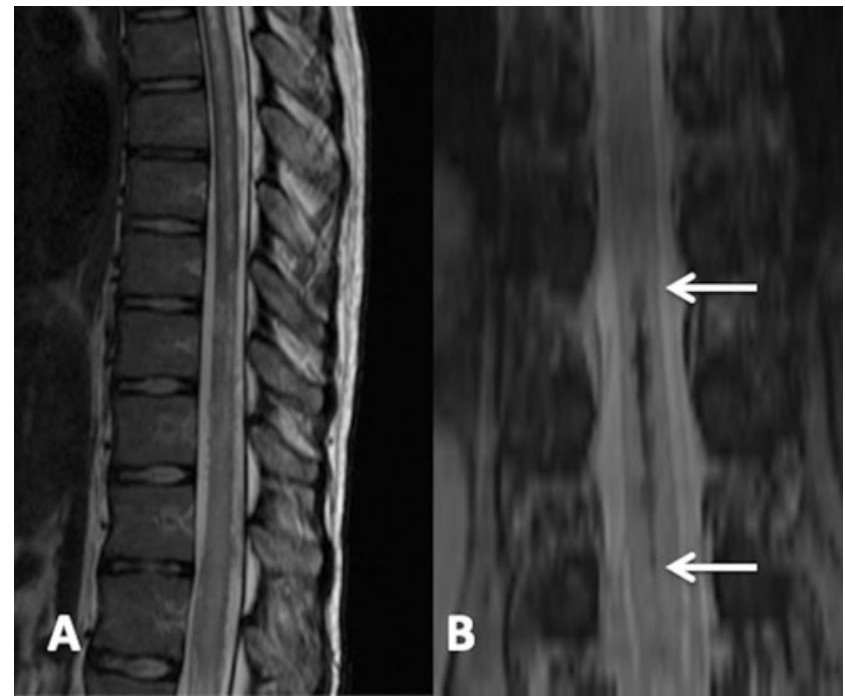

Fig. 2 Magnetic resonance images in the same patient. Sagittal T2weighted image. (a) Extensive T2 hyperintensity within the visualized lower thoracic cord extending to the conus medullaris. Coronal gradient-recalled-echo image (b) shows central hemorrhage within the lower thoracic cord (arrows) 
and death can be developed. Further research by radiologists will assist with this process.

Acknowledgments This research has been funded by a grant from the Royal Australian and New Zealand College of Radiology (RANZCR).

\section{References}

1. www.en.wikipedia.org/wiki/History_of_surfing.

2. http://www.surfline.com/community/whoknows/whoknows.cfm? id=1012. Accessed 29 October 2012

3. Dimmick S, Brazier D, Wilson P, Anderson SE. Injuries sustained to the spine while surfboard riding. Emerg Radiol. (2012, in press).

4. Nathanson A, Bird S, Dao L, Tam-Sing K. Competitive surfing injuries: a prospective study of surfing-related injuries among contest surfers. Am J Sports Med. 2007;35(1):113-7.

5. Taylor DM, Bennett D, Carter M, Garewal D, Finch CF. Acute injury and chronic disability resulting from surfboard riding. J Sci Med Sport. 2004;7(4):429-37.

6. Nathanson A, Haynes P, Galanis D. Surfing injuries. Am J Emerg Med. 2002;20(3):155-60.
7. Squire T, Sherlock M, Wilson P, Tan B, Hope N, Anderson SE. Surfinoma: a case report on a pseudotumour developing after a surfing sports injury. Skeletal Radiol. 2010;39(12):1239-43.

8. Thompson TP, Pearce J, Chang G, Madamba J. Surfer's myelopathy. Spine. 2004;29(16):E353-6.

9. Shuster A, Franchetto A. Surfer's myelopathy-an unusual cause of acute spinal cord ischemia: a case report and review of the literature. Emerg Radiol. 2011;18(1):57-60.

10. Aviles-Hernandex I, Garcia-Zozaya I, De Villasante JM. Nontraumatic myelopathy associated with surfing. J Spinal Cord Med. 2007;30 (3):288-93.

11. Fessa CK, Lee BS. An Australian case of surfer's myelopathy. Clin J Sport Med. 2012;22(3):281-3.

12. Chung HY, Sun SF, Wang JL, Lai PH, Hwang CW. Non-traumatic anterior spinal cord infarction in a novice surfer: a case report. J Neurol Sci. 2011;302(1-2):118-20.

13. Dhaliwal P, Cenic A, Eesa M, du Plessis S. An unusual case of myelopathy: surfer's myelopathy. Can J Neuro Sci. 2011;38 (2):354-6.

14. Karabegovic A, Strachan-Jackman S, Carr D. Surfer's myelopathy: case report and review. CJEM. 2011;13(5):357-60.

15. Wright KM. A case of surfers' myelopathy. AJCM. 2010;7(2):74-5.

16. Lieske J, Cameron B, Drinkwine B, et al. Surfer's myelopathy demonstrated by diffusion-weighted magnetic resonance imaging: a case report and literature review. J Comput Assist Tomogr. 2011;35 (4):492-4. 